\title{
Geoquímica orgánica de biomarcadores en petróleos de las Cuencas del Golfo de San Jorge y Austral
}

\section{Organic geochemistry of biomarkers in oils from the Golfo de San Jorge and Austral Basins}

\author{
German Javier Tomas ${ }^{1,2}$, Adrián Javier Acuña ${ }^{2}$ \\ gquimico90g@hotmail.com,adrianjacuna@yahoo.com.ar \\ ${ }^{1}$ Centro de Investigación y Transferencia de la Provincia de Santa Cruz - CONICET. Av. \\ Lisandro de la Torre 860, Río Gallegos (9400) - Santa Cruz - Argentina. \\ ${ }^{2}$ Grupo de Estudios Ambientales (GEA) Universidad Tecnológica Nacional - Facultad \\ Regional Santa Cruz. Av. de los Inmigrantes 555, Río Gallegos (9400) \\ Santa Cruz - Argentina.
}

Recibido: 12/05/2020. Aceptado: 17/11/2020

\begin{abstract}
RESUMEN
El petróleo es una sustancia química compleja que constituye un recurso natural no renovable. Entre los compuestos que lo conforman contiene unas moléculas conocidas como biomarcadores. El objetivo del presente trabajo fue comparar las características paleoambientales de la Cuenca Austral (A) y del Golfo de San Jorge (GSJ) usando biomarcadores de muestras de petróleo de dichas cuencas productivas. Para tal fin, las muestras de crudo fueron sometidas a un abordaje experimental para la separación de sus fracciones alifáticas y aromáticas por cromatografía en columna de sílica gel y los extractos obtenidos fueron analizados por separado por cromatografía gaseosa con detección de masas estudiando los iones característicos de interés. Con los datos obtenidos se calcularon relaciones de diagnóstico (P/F, Ts/Tm, entre otros). Los resultados permitieron identificar diferencias significativas, la GJS indicó un aporte de materia orgánica Tipos I/II y II/III biodegradada bajo un ambiente de depositación anóxico, presencia de aguas de alta salinidad y una litología con influencia carbonática. Por otra parte, A sugirió crudos con un nivel de madurez térmica superior respecto GJS asociados a kerógenos tipo II/III con baja o nula biodegradación formados en un paleoambiente subóxico a partir de una roca madre siliciclástica con una importante presencia de arcillas. Este estudio demuestra que la conocida utilidad de los biomarcadores para definir paleoambientes e identificar procesos de biodegradación es aplicable para caracterizar las cuencas estudiadas, y que además permite discriminar entre los crudos de ambas cuencas.
\end{abstract}

Palabras clave: Condiciones paleoambientales; biodegradación; cromatografía.

\begin{abstract}
Petroleum is a complex chemical that is a non-renewable natural resource. Among the compounds that make it up, it contains molecules known as biomarkers. The objective of this work was to compare the paleoenvironmental characteristics of the Austral Basin (A) and the Gulf of San Jorge (GSJ) using biomarkers from oil samples from these productive basins. For this purpose, the crude oil samples were subjected to an experimental approach for the
\end{abstract}


separation of their aliphatic and aromatic fractions by silica gel column chromatography and the extracts obtained were analyzed separately by gas chromatography with mass detection studying the characteristic ions of interest. With the data obtained, diagnostic relationships (P/F, Ts/Tm, among others) were calculated. The results allowed identifying significant differences in the results of the biomarkers according to the basin from which the studied oil comes. The results obtained for the GJS indicate a contribution of organic matter Types I/II and II/III biodegraded under an anoxic deposition environment, presence of high salinity waters and a lithology with carbonate influence. On the other hand, the results of A suggest crude oils with a higher level of thermal maturity associated with type II/III kerogens with low or no biodegradation formed in a sub-toxic paleoenvironment from a siliciclastic bedrock with a significant presence of clays. This study shows that the well-known usefulness of biomarkers to define paleoenvironments and identify biodegradation processes is applicable to characterize the studied basins, and that it allows discriminating between crude oils from both basins.

Keywords: Paleoenvironmental conditions; biodegradation; chromatography.

\section{INTRODUCCIÓN}

Los biomarcadores son moléculas complejas presentes en el petróleo y que se caracterizan por su elevada estabilidad química durante los procesos que le dieron origen (diagénesis, catagénesis y metagénesis), es decir, son aquellos compuestos que pueden ligarse de forma inequívoca con sus precursores biológicos ya que el esqueleto de carbonos se preserva (Peters et al., 2005). En comparación con las concentraciones de los precursores biogénicos en los sedimentos, las concentraciones de biomarcadores en el petróleo son bajas, a menudo no alcanzando las cien partes por millón. Sin embargo, pueden ser detectados a pesar de la presencia de una amplia variedad de otros tipos de hidrocarburos en el petróleo mediante el uso de la espectrometría de masas acoplada a la cromatografía de gases (GC-MS) (Stashenko et al., 2014).

El petróleo se puede separar en fracciones: alifática, aromática, resina y asfáltenos. En cada una de ellas están presentes los biomarcadores, pero las resinas y los asfáltenos no se volatilizan por debajo de $350^{\circ} \mathrm{C}$ por lo que sólo se trabaja con los biomarcadores presentes en las fracciones más livianas del crudo (Stashenko et al., 2014). El petróleo tiene una composición variable la cual depende de la roca madre que lo generó, del régimen térmico que predominó durante su formación, de la migración geológica y de las condiciones del yacimiento (Peters et al., 1993). La importancia del conocimiento geoquímico radica en la gran utilidad que tienen los biomarcadores para detectar las condiciones paleoambientales en que ocurre su depositación, ya que permite identificar las características del maceral (Rangel et al., 2017). Cada crudo en parte es un reflejo de la clase de materia orgánica que se depositó con los sedimentos que conforman la roca madre, así como de los procesos físicos-químicos a los que fueron sometidos desde la sedimentación hasta la maduración térmica de la roca madre (Rangel et al., 2017). Estas combinaciones de moléculas, con sus respectivas reacciones químicas son únicas y varían dependiendo de la cuenca y/o campo, por lo que son indicadores de ubicaciones específicas. Además, permiten sugerir la madurez térmica, el tipo de litología de la roca generadora y el aporte de material orgánico. En muchos casos las acumulaciones de petróleo son descubiertas antes que las rocas que les dieron génesis. En estos casos es muy útil comparar la geoquímica orgánica del petróleo con los extractos geoquímicos de una muestra de su potencial roca generadora, y vincularlo (o no) a su fuente (Zhang et al., 2015). 
Los biomarcadores más estudiados debido a la facilidad con la que se determinan por las técnicas analíticas son el Pristano $(\mathrm{P})$ y el Fitano $(\mathrm{F})$, compuestos alifáticos ramificados que derivan de la molécula de fitol asociada a la estructura de la clorofila (Killops y Killops, 2005). Otros compuestos son los hopanos, de naturaleza policíclica que pueden presentar aromaticidad en algunos casos. Su presencia está relacionada con los ácidos hopanoides de las bacterias. Un tercer grupo son los esteranos, también policíclicos pero menos complejos que los hopanos, que derivan de los esteroles de células eucariotas (Philp y Oung, 1988). Los hopanos y los esteranos se identifican en espectrometría de masas mediante los iones $\mathrm{m} / \mathrm{z}=$ $191 \mathrm{y} \mathrm{m} / \mathrm{z}=217$ respectivamente. Además de los biomarcadores, en los estudios geoquímicos orgánicos también se analiza la presencia y concentración de otras moléculas conocidas como marcadores aromáticos que complementan la información suministrada. A este grupo pertenecen los naftalenos, fenantrenos, dibenzotiofenos, entre otros (Silvan et al., 2008). En este trabajo presentamos un abordaje experimental para caracterizar geoquímicamente los petróleos de las cuencas Austral (A) y Golfo de San Jorge (GSJ). El análisis realizado de los biomarcadores permite a los geoquímicos del petróleo caracterizar crudos en términos de (i) condiciones paleoambientales, (ii) madurez térmica, (iii) fuentes biológicas de materia orgánica sedimentaria, y (iv) evaluar la migración y el grado de biodegradación basado en la pérdida de alcanos, isoprenoides, compuestos aromáticos, terpanos y esteranos (Rangel et al., 2017).

La República Argentina tiene en su artículo 41 de la constitución nacional (CN) el mandato de preservar el medio ambiente para que todos los habitantes gocen del derecho a un ambiente sano, equilibrado, apto para el desarrollo humano y para que las actividades productivas satisfagan las necesidades presentes sin comprometer las de las generaciones futuras. Este mandato de la $\mathrm{CN}$ luego propulsa la generación de numerosas leyes nacionales que buscan establecer los presupuestos mínimos necesarios para preservar el medioambiente y sus reglamentaciones, así como el marco normativo provincial y/o municipal, definen parámetros ambientales que sirven para detectar cuando se ocasiona daño ambiental y cómo debe obrarse para su restitución a través de las resoluciones que impartan las autoridades de aplicación competentes. En el caso particular de las situaciones ambientales con afectaciones de petróleo y/o sus derivados, la implementación de las técnicas que se presentan en este trabajo pueden ser de gran utilidad para establecer la trazabilidad de la fuente de afectación brindando información muy específica y de gran importancia para las investigaciones forenses medioambientales en términos de determinar el origen de los hidrocarburos derramados, la diferenciación y la correlación de los petróleos, el comportamiento de los hidrocarburos en el medio ambiente y el seguimiento del proceso de degradación a la intemperie en una amplia variedad de condiciones ambientales (Zhang et al., 2015). Otras aplicaciones geoquímicas forenses de estas metodologías evocadas pueden extenderse a (i) la comercialización fraudulenta de crudos y derivados porque permite identificarlos bajo condiciones de procedencia dudosa y/o incautamiento por sospecha de hurto y (ii) conocer el rendimiento de los distintos métodos de degradación de los residuos del petróleo, principalmente los que se refieren el empleo de microorganismos. Asimismo, se puede evaluar la eficacia del uso de diferentes fertilizantes o catalizadores que intervienen en la proliferación de las bacterias y, en consecuencia, en la degradación de los productos del petróleo (Yim et al., 2011).

Las cuencas sedimentarias son ámbitos de la corteza terrestre que tienen un comportamiento subsidente dando contextos geotectónicos específicos en donde se acumulan sedimentos. Estas zonas en casos de darse condiciones favorables permiten la formación y acumulación de hidrocarburos. Las cuencas de estudio, A y GSJ, están ubicadas en la porción meridional de la Patagonia. La cuenca GSJ es de tipo intracratónica, predominantemente extensional, con una orientación general Este-Oeste, se extiende desde proximidades de Río Mayo (Chubut) hasta dentro del Golfo de San Jorge en el Mar Argentino; su basamento está compuesto por un 
complejo volcánico-sedimentario jurásico (Uliana y Lagarreta, 1999) y posee dos rocas madres conocidas, la de la Fm. Anticlinal Aguada Bandera y la de la Fm. Pozo D-129, que constituyen entonces dos sistemas petroleros diferentes (Figari et al., 1999). La historia geológica de la Cuenca Austral está relacionada a tres etapas tectónicas principales (Biddle et al., 1986; Robbiano et al., 1996; Ramos, 2002; Kraemer et al., 2003; Peroni et al., 2002; Rodríguez y Miller, 2005). Éstas son la etapa de rift, la etapa de hundimiento termal con el desarrollo de una cuenca marginal (Cuenca Marginal de Rocas Verdes) y por último la etapa de antepaís. El principal sistema petrolero de la cuenca es el que se genera con la depositación de un ciclo sedimentario transgresivo que dio lugar a la depositación de la F. Palermo Aike Inferior compuesta de lutitas ricas en materia orgánica. Los reservorios típicos de la cuenca están representados por areniscas glauconíticas y de cuarzo y lutitas, depositadas en un entorno marino y conglomerados de areniscas, lutitas y lechos de lignito de los Miembros Inferior y Superior de la F. Magallanes (Villar y Arbé, 1993).

El petróleo de ambas cuencas ha sido frecuentemente caracterizado, pero existe una proporción menor de estudios centrados en el perfil de biomarcadores de dichos petróleos. La presente contribución busca mejorar entonces el grado de conocimiento existente sobre las condiciones en que se generaron los petróleos en ambas cuencas a través del uso de las características de sus biomarcadores.

\section{Objetivo}

El objetivo del presente trabajo fue determinar los biomarcadores presentes en cuatro crudos, de los cuales dos pertenecen a la Cuenca Austral y los otros dos a la Cuenca del Golfo de San Jorge. Una vez obtenidos los resultados analíticos, evaluar las características que se pueden deducir de los mismos para poder identificar diferencias en la generación de estos crudos y sus cuencas.

\section{Muestras}

\section{MATERIALES Y MÉTODOS}

Para la realización del presente trabajo, se obtuvieron dos muestras crudo pertenecientes a la Cuenca Austral y dos muestras de la Cuenca del Golfo de San Jorge. Las muestras denominadas como COA1 y COA2 fueron tomadas de oleoductos de la Cuenca Austral. COA1 fue colectada del denominado Oleoducto Este que recoge la producción de los yacimientos La Maggie y Cañadón Salto, mientras que COA2 del denominado Oleoducto Oeste que recoge la producción de los yacimientos Dos Hermanos, Campo Boleadoras, Campo Indio Este y María Inés. Las muestras denominadas COG3 y COG4 fueron colectadas en oleoductos de la Cuenca del Golfo de San Jorge. COG3 se tomó del tramo del oleoducto oeste-este de la zona norte de la Provincia de Santa Cruz antes de su llegada a las instalaciones de almacenamiento en la ciudad de Caleta Olivia y que recoge la producción de los yacimientos de Las Heras, Piedra Clavada, Cañadón Seco y Cañadón León, mientras que COG4 se colectó del tramo del oleoducto de la Planta Km 9 de la ciudad de Comodoro Rivadavia próximo a intersectar con el oleoducto oeste-este de la Provincia de Chubut y de confluir a las instalaciones de almacenamiento en Caleta Córdova y que recoge la producción de los yacimientos de Km 8, Don Ernesto y Don Alberto.

Las mismas se transportaron al laboratorio en botellas limpias de vidrio de un litro de capacidad color ámbar para evitar la fotooxidación. En el llenado de las botellas, se evitó la presencia de cámara de aire para minimizar el impacto del oxígeno en la estabilidad de las 
muestras, las que fueron almacenadas en lugares oscuros y secos a temperatura ambiente hasta la realización de los análisis.

\section{Acondicionamiento de las muestras de petróleo}

Aproximadamente $100 \mu \mathrm{L}$ de crudo se sometieron a una separación en columna de vidrio (20 $\mathrm{cm} \times 1,2 \mathrm{~cm}$ ) en sus fracciones alifáticas y aromáticas. Para este fin, se empacó la columna de vidrio con $3 \mathrm{~g}$ de gel de sílice activado, al que se le adicionó una columna de $50 \mathrm{mg}$ de sulfato de sodio activado y $50 \mathrm{mg}$ de alúmina activada en su parte superior. La mezcla de hidrocarburos se eluyó con $10 \mathrm{~mL}$ de n-pentano y $10 \mathrm{~mL}$ de diclorometano para obtener las fracciones alifáticas y aromáticas, respectivamente. Las resinas y los asfáltenos quedaron retenidos en la columna descartándose finalmente. Los extractos obtenidos se concentraron por separado hasta $0,5 \mathrm{ml}$ bajo corriente de nitrógeno y se transfirieron a un vial de cromatografía. Por último se almacenaron a $-15^{\circ} \mathrm{C}$ hasta su análisis (Leal et al., 2011).

\section{Análisis cromatográficos}

Se inyectó un microlitro de las fracciones alifáticas y aromáticas del crudo en modo splitless en un cromatógrafo de gases. La separación cromatográfica se realizó en un cromatógrafo de gases Agilent modelo 7890A, con detector por espectrometría de masas de la misma marca modelo 5975C. Se utilizó una columna HP5ms de $30 \mathrm{~m}$ de largo, con un diámetro interno de $0,32 \mathrm{~mm}$ y un espesor de película de $0,25 \mu \mathrm{m}$. La temperatura del inyector se ajustó a $290{ }^{\circ} \mathrm{C}$ y se utilizó helio como gas portador con una velocidad de flujo de $1,2 \mathrm{~mL} \cdot \mathrm{min}^{-1}$. El programa de temperatura utilizado fue el siguiente: temperatura inicial de $55{ }^{\circ} \mathrm{C}$ durante $2 \mathrm{~min}$, seguido de una rampa de $6{ }^{\circ} \mathrm{C} \cdot \mathrm{min}^{-1}$ hasta alcanzar los $270{ }^{\circ} \mathrm{C}$, pasando directamente a otra rampa de $3{ }^{\circ} \mathrm{C} \cdot \mathrm{min}^{-1}$ hasta alcanzar $300{ }^{\circ} \mathrm{C}$, temperatura que se mantuvo durante $17 \mathrm{~min}$. El tiempo total de corrida fue de aproximadamente $65 \mathrm{~min}$. El detector de masas se utilizó con una temperatura de la fuente de iones y de la línea de transferencia de $230{ }^{\circ} \mathrm{C}$ y $180{ }^{\circ} \mathrm{C}$ respectivamente y una energía de impacto de $70 \mathrm{eV}$. El escaneo de masas entre 30 y 400 uma se realizó en modo Scan para analizar los iones $\mathrm{m} / \mathrm{z}=178, \mathrm{~m} / \mathrm{z}=184, \mathrm{~m} / \mathrm{z}=192, \mathrm{y} \mathrm{m} / \mathrm{z}=198$ de la fracción aromática, y en modo SIM para los iones $\mathrm{m} / \mathrm{z}=191 \mathrm{y} \mathrm{m} / \mathrm{z}=217$ para el extracto de la fracción alifática (Stashenko et al., 2014).

\section{Análisis de datos}

Al momento de procesar toda la información obtenida en un estudio de biomarcadores, es decir, analizar la presencia/ausencia y concentración de estos en las muestras analizadas, los resultados más importantes derivan de las Relaciones de Diagnóstico. Para obtener estas relaciones se debe tomar uno o más biomarcadores pertenecientes a un grupo en particular y dividirlos con uno o más biomarcadores de otro o del mismo grupo. Con este procedimiento, aunque las concentraciones independientes de los biomarcadores varíen, las relaciones de diagnóstico permanecen constantes lo que permite entre otras cosas contrastar muestras. Para esto, se estudiaron los compuestos $\mathrm{P}, \mathrm{F}$ y n-alcanos a partir de los extractos de petróleo, los hopanos y esteranos a partir de los extractos de las fracciones alifáticas, y los compuestos de tipo aromáticos a partir de los análisis cromatográficos de los extractos aromáticos. A continuación, se detallan algunas relaciones de diagnóstico utilizadas por los geoquímicos orgánicos y que fueron utilizadas en este estudio mediante las ecuaciones 1 y 2. 


$$
\begin{gathered}
P / F=\text { Pristano } / \text { Fitano } \\
T s+T m / \text { Ts }=\text { Trisnorneohopano }+ \text { Trisnorhopano } / \text { Trisnorneohopano }
\end{gathered}
$$

\section{RESULTADOS Y DISCUSIÓN}

\section{Materia orgánica precursora}

La relación $\mathrm{P} / \mathrm{F}$ y la que se obtiene de ellos con sus alcanos normales $\left(\mathrm{n}-\mathrm{C}_{17}\right.$ y $\left.\mathrm{n}-\mathrm{C}_{18}\right)$ proporciona relevante información. Por ejemplo, permite inferir sobre el tipo de materia orgánica asociado a un crudo (Lo Mónaco et al., 1999). Los valores de la relación P/F (Cuadro 1) para COA1 y COA2 se ubicaron en torno a 2, lo cual permite inferir que la materia orgánica que dio lugar a estos petróleos es un kerógeno tipo II-III (Mixto). La sección generadora de la A (Inoceramus Inferior) se caracteriza por tener este tipo de materia orgánica según análisis realizados por Lagarreta y Villar (2011). En cambio, para COG3 y COG4, la relación $\mathrm{P} / \mathrm{F}$ fue aproximadamente 1 por lo que se puede sugerir la presencia de kerógenos tipo I/II (Lacustre/Marino) y II/III (Marino/Terrestre). Estudios previos realizados sobre petróleos pertenecientes al sistema petrolero D-129/Bajo Barreal indicaron la presencia de esta mezcla de materia orgánica (Villar et al., 1998). Sin embargo, este resultado por sí solo no puede ser utilizado para inferir el origen de la materia orgánica ya que la relación $\mathrm{P} / \mathrm{F}$ puede verse afectada por el nivel de madurez térmica del kerógeno, diferentes fuentes de materia orgánica (Tissot y Welte, 1984) o coelución de otros isoprenoides con el mismo tiempo de retención (Leythaeuser y Schwarzkopf, 1986).

\begin{tabular}{lcccc}
\hline RD & COA1 & COA2 & COG3 & COG4 \\
\hline $\mathrm{P} / \mathrm{F}$ & 1,81 & 2,05 & 1,06 & 0,88 \\
$\mathrm{P} / \mathrm{n}-\mathrm{C}_{17}$ & 0,52 & 0,29 & 0,40 & 1,66 \\
$\mathrm{~F} / \mathrm{n}-\mathrm{C}_{18}$ & 0,32 & 0,17 & 0,38 & 1,81 \\
$\mathrm{IMP}$ & 0,88 & 1,31 & 0,62 & 0,46 \\
$\mathrm{Rc}$ & 0,92 & 1,16 & 0,78 & 0,69 \\
$\mathrm{H}_{29} / \mathrm{H}_{30}$ & 0,84 & 0,87 & 1,04 & 1,07 \\
$\left(10 \times \mathrm{G}_{30}\right) /\left(\mathrm{G}_{30}+\mathrm{H}_{30}\right)$ & 4,32 & - & 13,61 & 14,14 \\
$\mathrm{Ts} / \mathrm{Tm}$ & 1,21 & 0,60 & 0,56 & 0,60 \\
$\mathrm{D}_{27} / \mathrm{S}_{27}$ & 1,23 & 0,83 & 0,42 & 0,30 \\
$\% \mathrm{~S}_{27}$ & 41,77 & 37,85 & 24,55 & 27,53 \\
$\% \mathrm{~S}_{28}$ & 29,22 & 35,65 & 31,71 & 28,97 \\
$\% \mathrm{~S}_{29}$ & 29,01 & 26,5 & 43,74 & 43,50 \\
\hline
\end{tabular}

Cuadro 1. RD: relación de diagnóstico, P/F:Prist/Fit ${ }^{1}, \mathrm{P} / \mathrm{n}-\mathrm{C}_{17}$ : Prist/Heptadecano ${ }^{2}, \mathrm{~F} / \mathrm{n}-\mathrm{C}_{18}$ : Fitano/Octadecano ${ }^{3}, \mathrm{Ts}_{28} / \mathrm{Ts}_{28}+\mathrm{Tm}_{27}$ : Trisnorneo hopano/Trisnorneohopano+Trisnorhopano ${ }^{4}, \quad \mathrm{H}_{29} / \mathrm{H}_{30}$ : Norhopano $\mathrm{C}_{29} / \mathrm{Hopano}_{30} \mathrm{C}^{5},\left(\begin{array}{lllll}10 & \mathrm{x} & \mathrm{G}_{30}\end{array}\right) /\left(\mathrm{G}_{30}+\quad \mathrm{H}_{30}\right):\left(10 \quad \mathrm{x} \mathrm{Gamacerano}_{30} \quad \mathrm{C}_{30}\right)$ $/\left(\text { Gamacerano } C_{30}+\text { Hopano } C_{30}\right)^{6}, M_{30} / H_{30}$ : Moretano $C_{30} /$ Hopano $C_{30}{ }^{7} \% S_{27}:\left(S_{27}(\alpha S+\alpha R+\beta S+\beta R) / S_{27}(\alpha S+\alpha R+\beta S+\beta R)+S_{28}(\alpha S+\right.$ $\left.\alpha \mathrm{R}+\beta \mathrm{S}+\beta \mathrm{R})+\mathrm{S}_{29}(\alpha \mathrm{S}+\alpha \mathrm{R}+\beta \mathrm{S}+\beta \mathrm{R})\right)^{8}, \% \mathrm{~S}_{28}:\left(\mathrm{S}_{28}(\alpha \mathrm{S}+\alpha \mathrm{R}+\beta \mathrm{S}+\beta \mathrm{R}) / \mathrm{S}_{27}(\alpha \mathrm{S}+\alpha \mathrm{R}+\beta \mathrm{S}+\beta \mathrm{R})+\mathrm{S}_{28}(\alpha \mathrm{S}+\alpha \mathrm{R}+\beta \mathrm{S}+\beta \mathrm{R})+\mathrm{S}_{29}(\alpha \mathrm{S}+\alpha \mathrm{R}\right.$ $+\beta S+\beta R))^{9}, \% S_{29}:\left(S_{29}(\alpha S+\alpha R+\beta S+\beta R) / S_{27}(\alpha S+\alpha R+\beta S+\beta R)+S_{28}(\alpha S+\alpha R+\beta S+\beta R)+S_{29}(\alpha S+\alpha R+\beta S+\beta R)\right)^{10} . S_{27}:$ Colestano, $\mathrm{S}_{28}$ : Ergostano, $\mathrm{S}_{29}$ : Estigmastano. ${ }^{1,2,3}$ Moldowan et al., 1994; ${ }^{4},{ }^{5},{ }^{6}$ Radke et al., $1988 ;{ }^{5}$ Peters y Moldowan, $1993 ;{ }^{4}$ McKirdy et al., 1983; ${ }^{6}$ Sinnighe Damsté et al., 1995; ${ }^{7}$ Seifert y Moldowan, 1980; ${ }^{8,9,10}$ Huang y Meinschein, 1976.

Los terpanos tricíclicos y los hopanos son los componentes más importantes del petróleo para determinar el tipo de materia orgánica, ya que sus precursores (terpenoides y hopanoides) conservan mejor la estructura inicial (Hunt, 1996). Estos se encuentran en la membrana celular de algas y procariotas respectivamente (Philp, 1985). En la Fig. 1 se observa una distribución similar para estos biomarcadores a nivel de cuenca, pero con importantes diferencias entre las cuencas. COG3 (Fig. 1C) y COG4 (Fig. 1D) presentaron como compuestos mayoritarios a los representados por los picos 1 y 2 asociados a terpanos tricíclicos que sugieren un aporte de algas por encima de las bacterias. Esto puede deberse a la depositación en zonas de alto nivel de la F. Pozo D-129 de pelitas oscuras y calizas oolíticas, y de clastos gruesos y material piroclástico en zonas de bajo nivel (Gómez Omil et al., 1990). 
Contrastando con lo antes mencionado, en COA1 (Fig. 1A) y COA2 (Fig. 1B) destacaron en los picos 7 y 8 (hopanos), que dieron lugar a una relación de materia bacterial/algal mayor a 1 . La cantidad de exinita (esporas, cutículas, algas) no supera el $20 \%$ en la composición de los kerógenos de la Cuenca Austral, mientras que las algas lacustres Botryococcus representan menos del $10 \%$ en estos kerógenos. En conclusión, la materia orgánica en las lutitas de Springhill continental derivó principalmente de bacterias con una contribución marginal de algas (Pittion, 1991).

Finalmente, los esteranos no existen como tales en los organismos vivos, provienen de esteroles presentes en algas, animales y plantas superiores por lo que proporcionan información sobre la materia orgánica precursora (Mackenzie et al., 1982). La roca madre de la A es marina y los crudos COA1 y COA2 exhibieron esa influencia debido al predominio del esterano $\mathrm{S}_{27}$ (Cuadro 1) presente en zooplancton, algas rojas y verdes (Moldowan et al., 1985). COG3 y COG4 sugirieron un marcado aporte de material terrígeno a la composición del crudo fundamentado por la presencia de $\mathrm{S}_{29}$ (Villar et al., 1996).

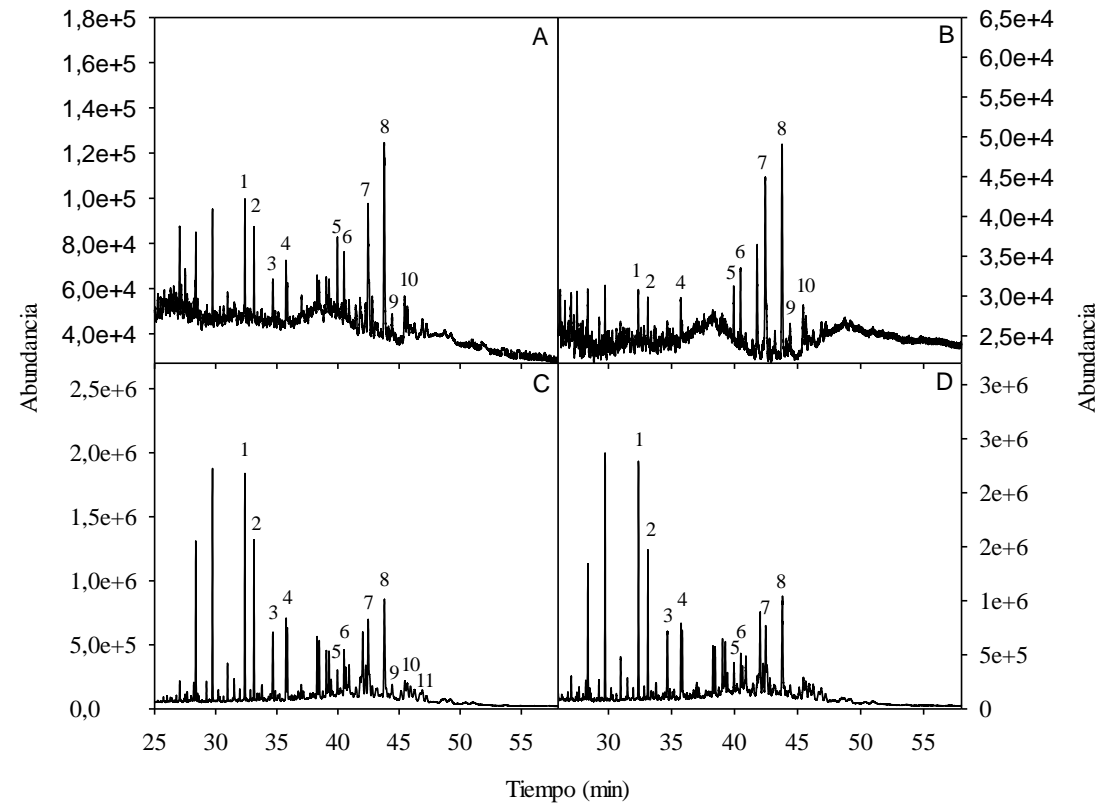

Figura 1. Fragmentogramas $\mathrm{m} / \mathrm{z}=191$ de las muestras de COA1 (A), COA2 (B), COG3 (C) y COG4 (D). En el eje de las ordenadas se observa la "abundancia relativa" y en el eje de las abscisas el "tiempo de retención". Terpanos (T) y hopanos $(H): 1\left(T_{23}\right), 2\left(T_{24}\right), 3\left(T_{25}\right), 4$ $\left(\mathrm{T}_{26}\right), 5(\mathrm{Ts}), 6(\mathrm{Tm}), 7\left(\mathrm{H}_{29}\right), 8\left(\mathrm{H}_{30}\right), 9\left(\mathrm{M}_{30}\right), 10\left(\mathrm{H}_{31}\right)$ y $11\left(\mathrm{G}_{30}\right)$.

\section{Ambiente de depositación y litología de la roca generadora}

La relación $\mathrm{P} / \mathrm{F}$ es un parámetro importante debido a que permite establecer el ambiente de depositación en el que fue sedimentada la materia orgánica (Peters et al., 1999). Para COA1 y COA2 se observó un valor de P/F tendiente a 2 (Cuadro 1), esto sugiere que las condiciones en las que se depositó la materia orgánica fueron subóxicas favoreciéndose la formación de $\mathrm{P}$ sobre F. La F. Springhill se caracteriza por una depositación transgresiva marino-litoral donde intercalan areniscas y arcillas en un ambiente subóxico (Lagarreta y Villar, 2011). En el caso de COG3 y COG4, los resultados cercanos a 1 se asociaron a condiciones con menor presencia de oxígeno inferidas por la presencia de un gran lago estratificado, relativamente somero relacionado a la megasecuencia II de la F. Pozo D-129 (Hechem et al., 1987).

A la hora de determinar la litología de la roca madre, los hopanos $\mathrm{H}_{29}$ y $\mathrm{H}_{30}$ deben tenerse en cuenta ya que son los hopanos dominantes (García et al., 1999). Los crudos de GSJ mostraron una influencia carbonática dada por valores de $\mathrm{H}_{29} / \mathrm{H}_{30}$ superiores a 1 asociados a niveles oolíticos (Rodríguez, 1997). COA1 y COA2 (Cuadro 1) pusieron de manifiesto una litología 
siliclástica para la roca generadora que es característica de A (Cagnolatti et al., 1995). Otro indicador a tener en cuenta es el que resulta de la relación entre el Gamacerano $\left(\mathrm{G}_{30}\right)$ y el hopano $\left(\mathrm{H}_{30}\right)$. El $\mathrm{G}_{30}$ se encuentra en concentraciones muy bajas en crudos y su presencia se relaciona a la elevada productividad bacteriana dentro de una columna de agua estratificada por salinidad o temperatura (Sinninghe-Damsté et al., 1995). COA1 y COA2 presentaron baja concentración de $\mathrm{G}_{30}$, sin embargo, fluctuaciones en los grados de hipersalinidad son característicos de la megasecuencia II de la GSJ (Gómez Omil et al., 1990) que se reflejaron en el índice de $\mathrm{G}_{30}$ por encima del $10 \%$ para COG3 y COG4.

Por último, $\mathrm{D}_{27} / \mathrm{S}_{27}$ refleja el contenido de arcilla presente en una roca madre. Las arcillas catalizan la transformación de esteranos a diasteranos que puede observarse en los fragmentogramas de la Fig. 2 (Lerch et al., 2016). El sistema petrolero Pozo D-129/Bajo Barreal de la GSJ exhibió un bajo contenido en arcillas debido a prevalencia de $\mathrm{S}_{27}$ sobre $\mathrm{D}_{27}$ (Cuadro 1). Por el contrario, los petróleos COA1 y COA2 destacaron por concentraciones importantes de arcillas en las litofacies de A.

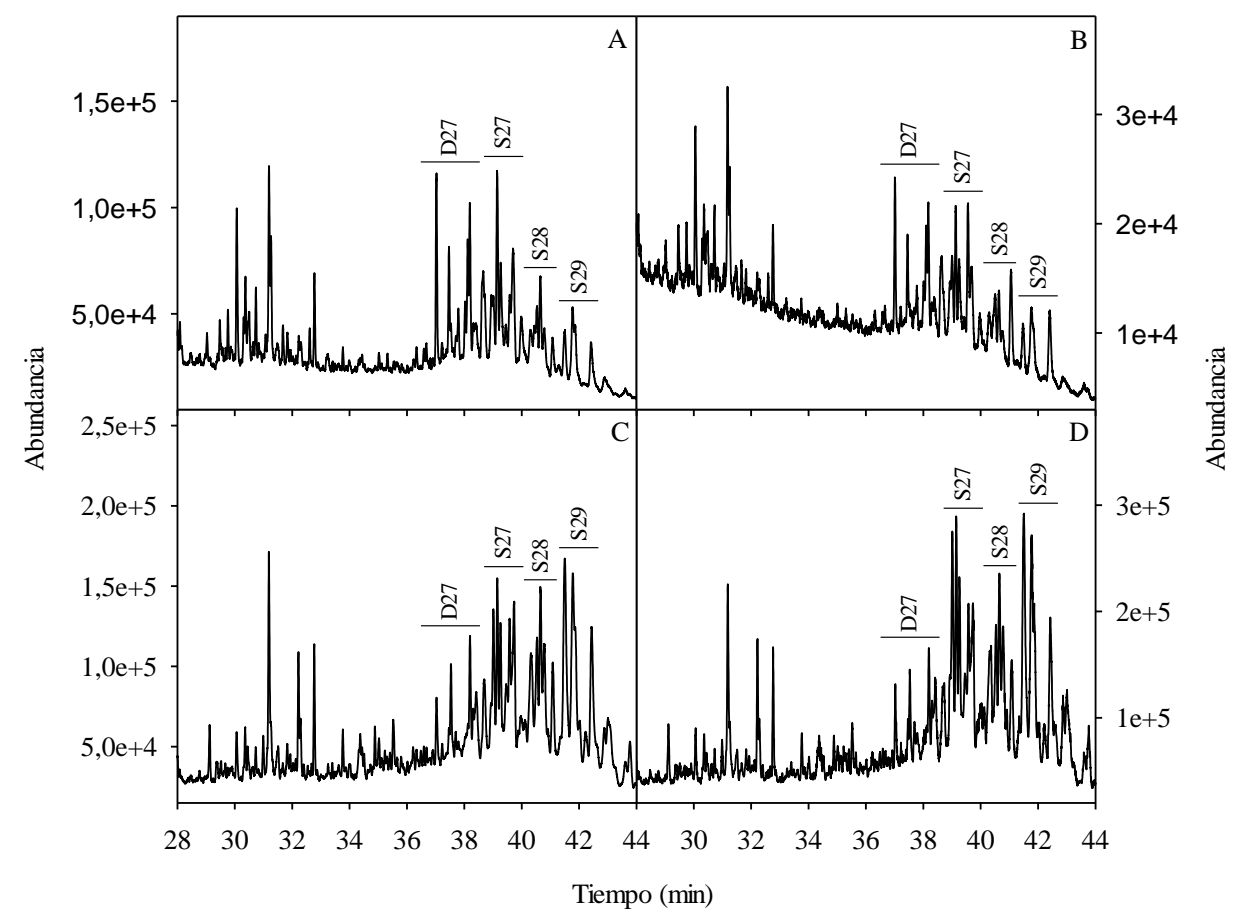

Figura 2. Fragmentogramas $\mathrm{m} / \mathrm{z}=217$ de las muestras de COA1 (A), COA2 (B), COG3 (C) y COG4 (D). En el eje de las ordenadas se

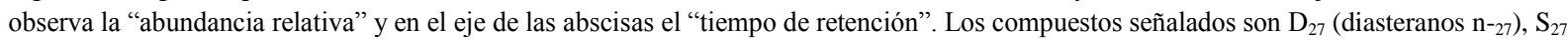
(colestanos), $\mathrm{S}_{28}$ (ergostanos) y $\mathrm{S}_{29}$ (estigmastanos).

\section{Madurez térmica}

Uno de los parámetros utilizados para determinar el nivel de madurez térmica de un crudo es el índice de metilfenantreno (IMP) que se basa en la distribución de metilfenantreno (MP) y sus hómologos metilados (Radke et al., 1986). Éstos pueden derivar de esteroides y triterpenoides del material biológico de partida, o bien pueden originarse a partir de reacciones de metilación del fenantreno (Killops y Killops, 1993). Los isómeros más estables desde el punto de vista termodinámico son el 2-MP y 3-MP frente al 1-MP y 9-MP (Nabbefeld et al., 2010). Por otro lado, la Reflectancia de Vitrinita Calculada (Rc) constituye la variable más importante al momento de determinar la evolución térmica de un crudo y se calcula a partir del IMP (Radke et al., 1986). La Rc mostró mayores valores para COA1 y COA2 (Cuadro 1) relacionado con una mayor evolución térmica de los crudos. Por su parte cabe destacar que $\mathrm{Tm}\left(\mathrm{C}_{27} 17 \alpha(\mathrm{H})\right.$-trisnorhopano $)$ y $\mathrm{Ts}\left(\mathrm{C}_{27} 18 \alpha(\mathrm{H})-22,29,30-\right.$ 
trisnorneohopano) son dos hopanos ampliamente utilizados para determinar madurez térmica. Tm es térmicamente más inestable y a medida que la madurez térmica aumenta, este se convierte en Ts por lo que bajos valores de la relación Ts/Ts + Tm es indicativo de crudos inmaduros (Seifert y Moldowan, 1978). COA1 tuvo una relación Ts/Ts + Tm muy alta (Cuadro 1), y el resto de las muestras valores por debajo y semejantes entre sí. Como cada una de las muestras es una mezcla de crudos y además no pertenecen a la misma facie orgánica, este parámetro no se puedo aplicar.

\section{Biodegradación}

El cromatograma de iones totales (TIC) mostró diferencias para los crudos estudiados (Fig. 3). En COG3 (Fig. 3C) apareció un levantamiento de la línea de base a partir del minuto 25 (tiempo retención) lo que pone de manifiesto el accionar de los microorganismos. COG4 (Fig. 3D) es un petróleo fuertemente biodegradado o paleobiodegradado debido a que presentó una mezcla compleja no resuelta (UCM) que es evidente en la Fig. 3, sumado a valores de $\mathrm{P} / \mathrm{C}_{17} \mathrm{y}$ $\mathrm{F} / \mathrm{C}_{18}$ por encima de uno (Cuadro 1). Esta característica fue documentada por Villar et al. (1996), concluyendo que los hidrocarburos estudiados resultaron de procesos complejos de biodegradación y mezcla, activos a lo largo de extensos períodos del tiempo geológico. Sus evidencias cromatográficas y moleculares les indicaron que las acumulaciones responden a mezclas de petróleos intensamente biodegradados (del tipo "tar-sand") que han sido generados y alterados en una etapa temprana y subsecuentemente mezclados con petróleos frescos de nueva generación y/o remigrados. En el caso de COA1 (Fig. 3A) y COA2 (Fig. 3B) no se observó un levantamiento de la línea de base, además los valores de las relaciones $\mathrm{P} / \mathrm{C}_{17}$ y $\mathrm{F} / \mathrm{C}_{18}$ están por debajo de 0,5 lo cual sugiere que los microorganismos no han degradado estos crudos.

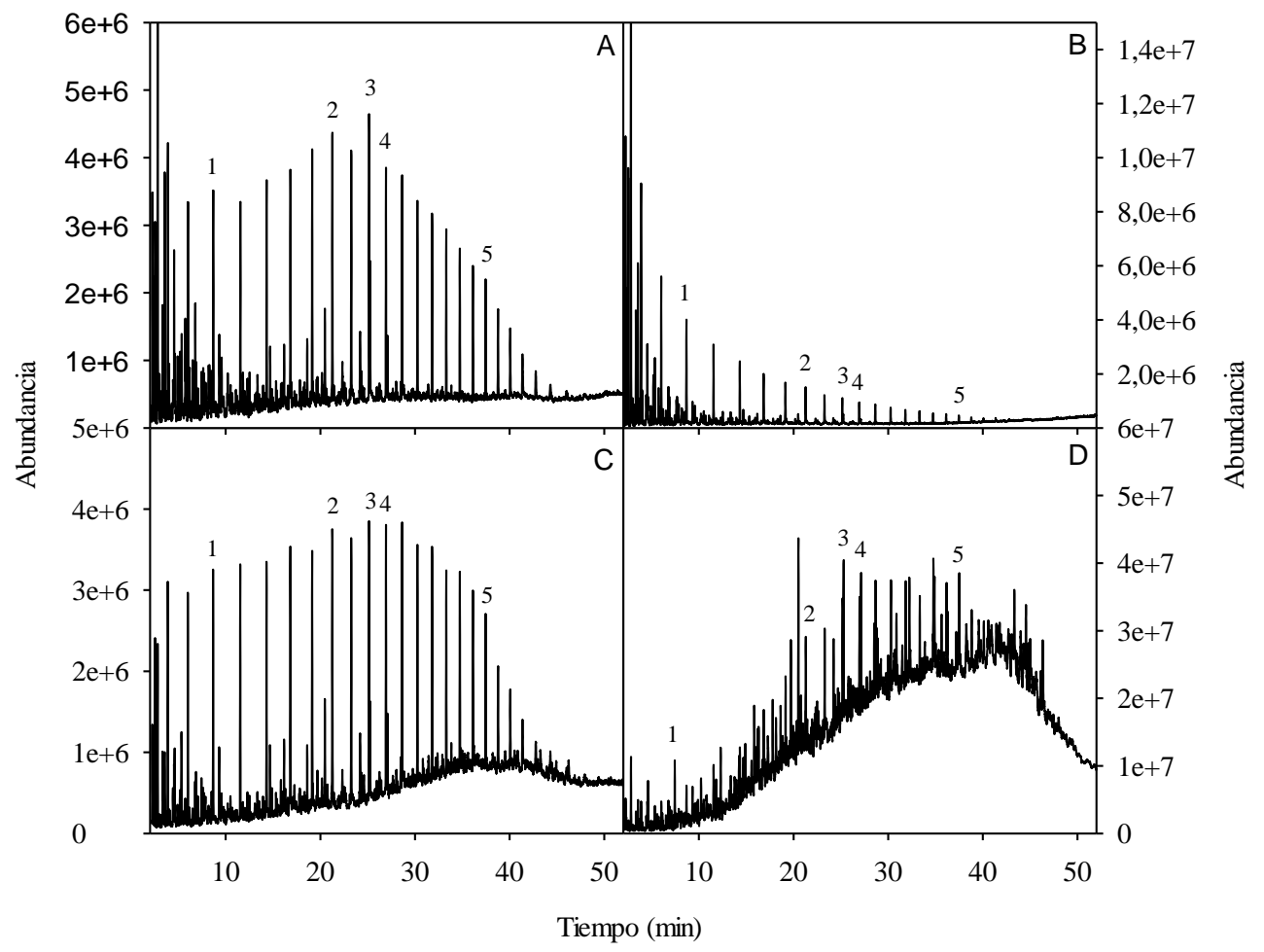

Figura 3. TIC para las muestras estudiadas de las muestras de COA1 (A), COA2 (B), COG3 (C) y COG4 (D). En el eje de las ordenadas se observa la "abundancia relativa" y en el eje de las abscisas el "tiempo de retención". Los compuestos señalados con números son los siguientes: $1\left(n-C_{10}\right), 2\left(n-C_{15}\right), 3\left(n-C_{17}\right), 4\left(n-C_{18}\right)$ y $5\left(n-C_{25}\right)$. 


\section{CONCLUSIONES}

Las relaciones de diagnóstico permiten sugerir que COA1 y COA2 se generaron a partir de kerógeno tipo II (marino/terrestre) no biodegradado y una roca madre siliclástica con presencia importante de arcillas bajo un ambiente subóxico y una madurez térmica superior respecto COG3 y COG4. Por otro lado, los crudos estudiados de la GSJ derivan de materia orgánica algal-lacustre predominante y contribución terrígena variable (Tipo I/II y Tipo II/III) biodegradada y una roca generadora con influencia carbonática en presencia de hipersalinidad bajo un ambiente tendiente a la anoxicidad. Promover la realización de este tipo de estudios de caracterización geoquímica de los crudos propulsando el conocimiento del perfil biomarcadores, constituye una herramienta eficaz no solo para la caracterización integral de los yacimientos en estudio, sino también para el desarrollo de información que sirva como base forense para comparar crudos procedentes de diferentes yacimientos y más aún si proceden de cuencas diferentes.

\section{AGRADECIMIENTOS}

Los autores agradecen la contribución financiera de Universidad Tecnológica Nacional Facultad Regional Santa Cruz (PI MSUTNSC0007712) y del Consejo Nacional de Investigaciones Científicas y Técnicas (CONICET).

\section{BIBLIOGRAFÍA}

BIDDLE, K., ULIANA, M., MITCHUM, R., FITZGERALD, M., WRIGHT, R. (1986). The stratigraphic and structural evolution of central and eastern Magallanes Basin, Southern America. In Foreland basins (Allen, P.; Homewood, P.; editors). International Association of Sedimentologists, Special Publication 8: 41-61.

CAGNOLATTI, M. J., MARTINS, R., VILLAR, H. J. (1995). La formación Lemaire como probable generadora de hidrocarburos en el área Angostura, Provincia de Tierra del Fuego, Argentina. XIII Congreso Geológico Argentino y III Congreso de Exploración de Hidrocarburos, Actas I, 123-129. Buenos Aires, Argentina.

GARCIA, J., SÁNCHEZ, J., GUZMÁN, M. (1999). Esteranos y terpanos como marcadores biológicos en la prospección petrolera. Journal of the Mexican Chemical Society, 43:1-6.

GOMEZ OMIL, R., ARROYO, H., LAFFITTE, G., MELO, A. (1990). Anteproyecto exploratorio para el Sector Oriental del Flanco Sur. YPF S.A, informe inédito: p.31. C. Rivadavia

HECHEM, J., FIGARI, E., MUSACCHIO, E. (1987). Cuenca del Golfo San Jorge, hallazgo de la Fm. Pozo D-129. I.A.P., Petrotecnia, 28 (11): p. 13-15. Buenos Aires

HUNT, J. (1996). Petroleum Geochemistry and Geology: San Francisco, Freeman, 743 p.

KILlOPS, S. D., KILlOPS, V. J. (1993). An Introduction to Organic Geochemistry. Longman Scientific \& Technical, Essex, UK, 265 p.

KILLOPS, S. D., KILLOPS, V. J. (2005). Introduction to Organic Geochemistry. Blackwell Publishing. Second Edition, UK, 393 p. 
KRAEMER, P., PLOSZKIEWICZ, J. V., RAMOS, V. A. (2002). Estructura de la Cordillera Patagónica Austral entre los $40^{\circ}$ y $52^{\circ} \mathrm{S}$. In Geología y Recursos Naturales de Santa Cruz (Haller, M.J.; editor). In Congreso Geológico Argentino, No. 15. Relatorio: 353364. El Calafate.

LAGARRETA, L., VILLAR, H. (2011). Geological and Geochimical Keys of the Potential Shale Resources, Argentina Basins. Adapted from presentation at AAPG Geoscience Technology Workshop, "Unconventional Resources: Basics, Challenges, and Opportunities for New Frontier Plays," Buenos Aires, Argentina, June 26-28, 2011.

LEAL, B., FREITES, M., BRACHO, E., MARTÍNEZ, M., LÓPEZ, L. (2011). Evaluación de la fase estacionaria en la separación SARA de dos crudos pertenecientes a la Subcuenca de Barinas, Venezuela. Revista Técnica de la Facultad de Ingeniería Universidad del Zulia, 34: 203 - 211.

LERCH, B., KARLSEN, D., SELAND, R., BACKER-OWE, K. (2016). Depositional environment and age determination of oils and condensates from the Barents Sea. Petroleum Geoscience. doi:10.1144/petgeo2016-039

LEYTAHEUSER, D., SCHWARZKOPF, T. (1986). The pristane/n-heptadecane ratio as an indicator for recognition of hydrocarbon migration effects. Organic Geochemistry, 10:191-197.

LO MONACO, S., LÓPEZ, L., LABASTIDA, E. (1999). Correlación de crudos de la subcuenca de Barinas, Venezuela. Revista Latinoamericana de Geoquímica Orgánica, 5:47-56.

MACKENZIE, A., BRASSELL, S., EGLINGTON, G., MAXWELL, J. (1982). Chemical fossils: The geological fate of steroids. Science, 217, 491-504.

MOLDOWAN, J. M., SEIFERT, W. K., GALLEGOS, E. J. (1985). Relationship between petroleum composition and depositional environment of petroleum source rocks. American Association of Petroleum Geologists, 69:1255-1268.

NABBEFELD, B., GRICE, K., SCHIMMELMANN, A., SUMMONS, R., TROITZSCH, U., TWITCHETT, R. (2010). A comparison of thermal maturity parameters between freely extracted hydrocarbons (Bitumen I) and a second extract (Bitumen II) from within the kerogen matrix of Permian and Triassic sedimentary rocks. Organic Geochemistry, 41: 78 - 87.

PERONI, G., CAGNOlATTI, M., PEDRAZZINI, M. (2002). Cuenca Austral: marco geológico y reserva histórica de la actividad petrolera. In Simposio Rocas Reservorio de las Cuencas Productivas de la Argentina. (Schiuma, M.; Hinterwimmer, G.; Vergani, G.; editors). Congreso de Exploración y Desarrollo de Hidrocarburos, No. 5: 11-19. Mar del Plata.

PETERS, K. E., MOLDOWAN, J. M. (1993). The Biomarker Guide. Interpreting Molecular Fossils in Petroleum and Ancient Sediments. Prentice Hall, Englewood Cliffs, New Jersey, USA.

PETERS, K. E., FRASER, T. H., AMRIS, W., RUSTANTO, B., HERMANTO, E. (1999). Geochemistry of crude oils from eastern Indonesia. American Association of Petrology Geologists Bulletin, 83:1927-1942. 
Peters, K. E., WAlters, C. C., MOLDOWAN, J. M. (2005). The Biomarker Guide. Volume I. Biomarkers and Isotopes in the Environment and Human History, Second Edition, Cambridge University Press, Cambridge, UK.

PHILP, R. P. (1985). "Fossil Fuel Biomarkers". Applications and Spectra. Elsevier, Amsterdam.

PHILP, R., OUNG, J. (1988). Biomarkers: occurence, utility, and detection. Analytical Chemistry, 60:887-896.

PITTION, J. (1991). Source-Rocks and Oil Generation in the Austral Basin. En XIII Word Petroleum Congress. Buenos Aires, 1991, Proceedings, Vol. 2. Exploration and Production, John Wiley \& Sons Ltd.

RADKE, M., WELTE, D., WILLSCH, H. (1986). Maturity parameters based on aromatic hydrocarbons: Influence of the organic matter type. Organic Geochemistry, 10:51-63.

RAMOS, V. A. (2002). Evolución Tectónica. In Geología y Recursos Naturales de Santa Cruz (Haller, M.J.; editor). Congreso Geológico Argentino, No. 15, Relatorio: 365-390. El Calafate.

RANGEL, A., OSORNO, J., RAMIREZ, J., DE BEDOUT, J., GONZÁLEZ, J., PABÓN, J. (2017). Geochemical Assessment of the Colombian oils base on bulk petroleum properties and biomarker parameters. Marine and Petroleum Geology, 86: 1291 1309.

ROBBIANO, J. A., ARBE, H., BANGUI, A. (1996). Cuenca Austral Marina. In Geología y Recursos Naturales de la Plataforma continental Argentina (Ramos, V.A.; Turic, M.; editors). Congreso Geológico Argentino, No. 13, Relatorio, y Congreso de Exploración de Hidrocarburos, No. 3: 343-358. Buenos Aires.

RODRIGUEZ, J. (1997). Modelado Bidimensional Integrado de la Cuenca del Golfo San Jorge: Reconstrucción de la Generación, Migración y Acumulación de Hidrocarburos. YPF S.A., informe inédito: p.60. C. Rivadavia

RODRIGUEZ, J. F., MILLER, M. (2005). Cuenca Austral. In Frontera Exploratoria de la Argentina (Chebli G. et al.; editors). In Congreso de Exploración y Desarrollo de Hidrocarburos, No. 6: 308-323. Mar del Plata.

SEIFERT, W., MOLDOWAN, J. (1978). Aplication of steranes, triterpanes and monoaromatics to the maturation of crude oils. Geochimica et Cosmochimica Acta, 42:71-95.

SINNINGHE-DAMSTE, J., KENIG, F., KOSTER, J., LEEUW, J. (1995). Evidence for gammacerano as an indicator of water column stratification. Geochimica et Cosmochimica Acta, 59:1895-1900.

SIVAN, P., DATTA, G., SINGH, R. (2008). "Aromatic biomarkers as indicators of source, depositional environment, maturity and secondary migration in the oils of Cambay Basin, India”. Organic Geochemistry, 39, 1620-1630.

STASHENKO, E. E., MARTÍNEZ, J. R., ROBLES, M. (2014). "Extracción selectiva y detección específica de biomarcadores saturados de petróleo". Scientia Chromatographica. 6(4):251-268.

TISSOT, B., WELTE, D. (1984). Petroleum formation and occurrence. Springer-Verlag, New York, 699p. 
ULIANA, M., LEGARRETA, L., LAFFITTE G., VILLAR, H. (1999). "Estratigrafía y geoquímica de las facies generadoras de hidrocarburos en las cuencas petrolíferas Argentinas”, Petrotecnia, Año XL (3), p.12-25. VILLAR, H.J; Arbe, H.A. (1993). “Oil generation in the Esperanza Area, Austral Basin, Argentina". Third Latin American Congress on organic geochemistry.

VILlAR, H. J., SYLWAN, C., PLEIMlinG, A. G., MILlER, M., CASTAÑO, J. R., DOW, W. G. (1996). Formación de petróleos pesados a partir de procesos de biodegradación y mezcla en el sistema petrolero Pozo D-129- Cañadón Seco, Flanco Sur de la Cuenca del Golfo San Jorge, provincia de Santa Cruz, Argentina. XIII Congreso Geológico Argentino y III Congreso de Exploración de Hidrocarburos, Actas I: p. 223-242.

VILLAR, H. J., LAFFITTE, G., LEGARRETA, L. (1998). The source rocks of the Mesozoic petroleum systems of Argentina: a comparative overview on their geochemistry, paleoenvironments and hydrocarbon generation patterns. AAPG International Conf. and Exhib. Extended Abstracts: pp. 186-187. R. Janeiro.

YIM, U. H., HA, S. Y., AN, J. G., WON, J. H., HAN, J. M., HONG, S. H., KIM, M., JUNG, J. H., SHIM, W. J. (2011). Fingerprint and weathering characteristics of stranded oils after the Hebei Spirit oil spill, J. Hazard. Mater, 197: 60-69.

ZHANG, H., YIN, X., ZHOU, H., WANG, J., HAN, L. (2015). Weathering characteristics of crude oils from Dalian oil spill accident, China. Aqua Procedia, 3: 238-244. 